\title{
An estuarine-tuned quasi-analytical algorithm (QAA-V): assessment and application to satellite estimates of SPM in Galveston Bay following Hurricane Harvey
}

Ishan D. Joshi and Eurico J. D'Sa

Correspondence to: Eurico J. D’Sa (ejdsa@1su.edu)

The copyright of individual parts of the supplement might differ from the CC BY 4.0 License. 


\section{$\underline{\text { Section S1 }}$}

Chlorophyll-a profiles along with depth-dependent mass-specific IOPs are necessary to create Rrs using Hydrolight ${ }^{\circledR}$ four component case-2 models (S1) (Mobley and Sundman 2013; Sathyendranath et al., 1989; Kirk 1994; Bukata et. al., 1995),

$$
\begin{aligned}
& a_{t}(\lambda)=a_{w}(\lambda)+a_{\phi}(\lambda)+a_{N A P}(\lambda)+a_{g}(\lambda) \\
& b_{b t}(\lambda)=b_{b w}(\lambda)+b_{b \phi}(\lambda)+b_{b N A P}(\lambda)
\end{aligned}
$$

Synthetic chlorophyll concentrations [Chl] $(\mathrm{N}=730)$ were obtained with 73 values and ranged between 1 to $40 \mathrm{mg} \mathrm{m}^{-3}$ with 10 repetitions to simulate random variability as observed in natural waters. A total of 730 phytoplankton absorption spectra $\left(a_{\phi}\right)$ were generated using six groups containing 83 in situ normalized $\mathrm{a}_{\phi}$ spectra; random normalized spectrum selection among groups were based on modeled $\mathrm{a}_{\phi} 440$, and the following bio-optical models (Bricaud et al., 1995; Fischer and Fell 1999; Mobley 1994) (Fig. S1; Table S1),

$$
\begin{aligned}
& a_{\phi}^{\text {Normalized }}(\lambda)=\frac{a_{\phi}(\lambda)}{a_{\phi} 440} \\
& a_{\phi} 440=0.06 \times[\mathrm{Chl}]^{0.65} \times \Re(1,2) \\
& a_{\phi}^{*}(\lambda)=\frac{a_{\phi}(\lambda)}{[\text { Chl }]}
\end{aligned}
$$
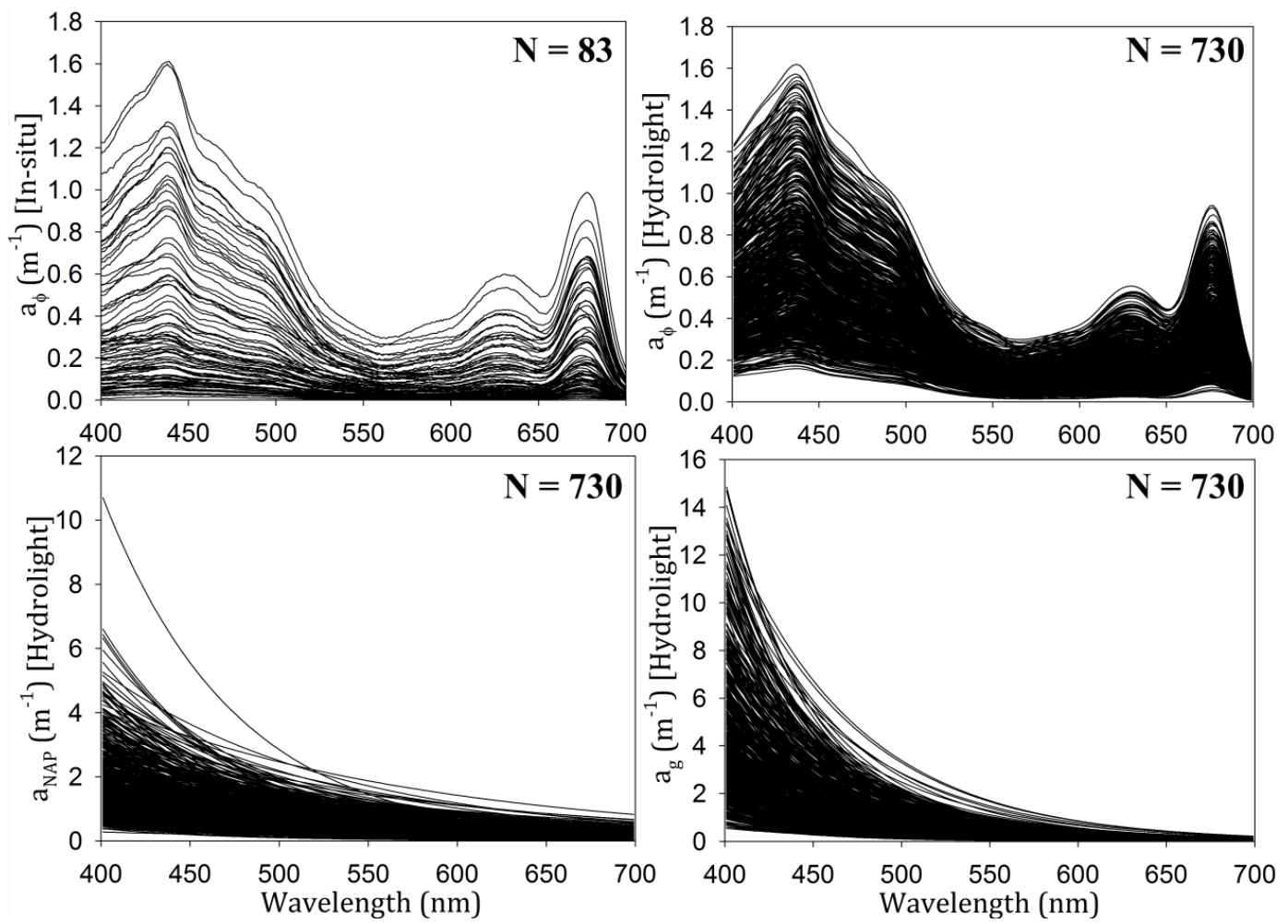

Figure S1. ( $\mathrm{a}, \mathrm{b})$ In situ phytoplankton absorption spectra $(\mathrm{N}=83)$ were collected from CDOM-dominated Apalachicola Bay (USA) and sediment-dominated Barataria Bay (USA) to generate 730 simulated spectra for Hydrolight ${ }^{\circledR}$ four-component Case-2 model using Eq. 2, (c) modeled non-algal particle absorption spectra $(\mathrm{N}=730)$ using Eq. 3, and (d) modeled CDOM absorption spectra $(\mathrm{N}=730)$ using $(\mathrm{S} 4)$.

Table S1: The conditions for allocating in-situ normalized-phytoplankton spectra $\left(a_{\phi}^{\text {normalized }}\right)$ into 6 groups. $\mathrm{a}_{\phi} 440$ is phytoplankton absorption at $440 \mathrm{~nm}$. 


\begin{tabular}{|c|c|}
\hline $\begin{array}{c}\text { Groups } \\
(\text { Total spectra }=83)\end{array}$ & Condition \\
\hline $1(\mathrm{n}=18)$ & $a_{\phi} 440<0.1$ \\
\hline $2(\mathrm{n}=15)$ & $a_{\phi} 440 \geq 0.1 \& a_{\phi} 440<0.2$ \\
\hline $3(\mathrm{n}=12)$ & $a_{\phi} 440 \geq 0.2 \& a_{\phi} 440<0.3$ \\
\hline $4(\mathrm{n}=11)$ & $a_{\phi} 440 \geq 0.3 \& a_{\phi} 440<0.5$ \\
\hline $5(\mathrm{n}=16)$ & $a_{\phi} 440 \geq 0.5 \& a_{\phi} 440<1.0$ \\
\hline $6(\mathrm{n}=11)$ & $a_{\phi} 440 \geq 1.0$ \\
\hline
\end{tabular}

Mass-specific non-algal particle absorption coefficients $\left(a_{\mathrm{NAP}}^{*}(\lambda)\right)$ were obtained with an exponential model (Roesler et al. 1989; Bricaud et al. 1995), where slope $\left(\mathrm{S}_{\mathrm{NAP}}\right)$ used a random value between $0.005-0.015$ as observed in natural waters ranging from oceanic to estuarine environments (Kirk 1994). The required $a_{N A P} 440$ was modeled using $a_{\phi} 440$ based on a constant $p_{1}\left(=a_{N A P} 440 / a_{\phi} 440\right)$ which was set to lie between 1 and 3.5 as generally observed in the two bays, e.g., $p_{1}=1$ represents pigment/CDOM-rich waters and $\mathrm{p}_{1}=3.5$ represents sediment/CDOM-rich waters (S3).

$$
\begin{aligned}
& \mathrm{a}_{\mathrm{NAP}}(\lambda)=\mathrm{a}_{\mathrm{NAP}} 440 \times \mathrm{e}^{-\mathrm{S}_{\mathrm{NAP}} \times(\lambda-440)} \\
& \mathrm{p} 1=1+\frac{2.5 \times \mathrm{a}_{\phi} 440 \times \Re(0,1)}{0.05+\mathrm{a}_{\phi} 440} \\
& \mathrm{a}_{\mathrm{NAP}}^{*}(\lambda)=\frac{\mathrm{a}_{\phi}(\lambda)}{[\mathrm{NAP}]} \\
& {[\mathrm{NAP}]=\mathrm{p} 1 \times[\mathrm{Chl}]}
\end{aligned}
$$

CDOM absorption coefficient was modeled with an exponential model (Bricaud et al. 1981), where spectral slope $\left(\mathrm{S}_{\mathrm{g}}\right)$ was chosen randomly between 0.01 to 0.025 as generally observed in various oceanic to productive estuarine environments (Kirk 1994; Babin et al., 2003) (Fig. S1d). $a_{g} 440$ was estimated from $\mathrm{a}_{\phi} 440$ and a constant $\mathrm{p} 2\left(=\mathrm{a}_{\mathrm{g}} 440 / \mathrm{a}_{\phi} 440\right) . \mathrm{p}_{2}$ was set between 1 to 4.5 based on field data, e.g., $\mathrm{p}_{2}=1$ represents phytoplankton/sediment dominated waters and $\mathrm{p}_{2}=4.5$ represents CDOM-rich waters (S4).

$$
\begin{aligned}
& \mathrm{a}_{\mathrm{g}}(\lambda)=\mathrm{a}_{\mathrm{g}} 440 \times \mathrm{e}^{-\mathrm{S}_{\mathrm{g}} \times(\lambda-440)} \\
& \mathrm{a}_{\mathrm{g}} 440=\mathrm{p} 2 \times \mathrm{a}_{\phi} 440 \\
& \mathrm{p} 2=1+\frac{3.5 \times \mathrm{a}_{\phi} 440 \times \Re(0,1)}{0.02+\mathrm{a}_{\phi} 440} \\
& \mathrm{a}_{\mathrm{g}}^{*}(\lambda)=\frac{\mathrm{a}_{\mathrm{g}}(\lambda)}{[\mathrm{Chl}]}
\end{aligned}
$$

Mass-specific phytoplankton backscattering $\left(\mathrm{b}_{\mathrm{b} \phi}^{*}\right)$ and non-algal particle back-scattering $\left(\mathrm{b}_{\mathrm{bNAP}}^{*}\right)$ coefficients were obtained based on the oceanic models (IOCCG 2006; please see references therein) with no change, as insufficient observations were available for estuarine environments. Subsequently, these coefficients were then converted to respective back-scattering coefficients by multiplying phase function dependent values "0.005" and "0.0183" (S5 and S6) (Mobley 1994; Mobley and Sundman 2013),

$$
\begin{aligned}
& \mathrm{b}_{\mathrm{bNAP}}^{*}(\lambda)=\frac{\mathrm{b}_{\mathrm{NAP}}(\lambda) \times 0.0183}{[\mathrm{NAP}]} \\
& \mathrm{b}_{\mathrm{b} \phi}^{*}(\lambda)=\frac{\mathrm{b}_{\phi}(\lambda) \times 0.005}{[\mathrm{Chl}]}
\end{aligned}
$$

Hydrolight ${ }^{\circledR}$ simulations were then run with a case-2 model to generate Rrs using mass-specific IOPs, chlorophyll concentrations, dark bottom sediments, finite depth of 5 meters, sun zenith angle of $30^{\circ}$, no Raman scattering, and no chlorophyll fluorescence. A total of 169 erroneous spectra were suspected 
possibly due to atypical combinations of $\mathrm{CDOM}$, non-algal particles, and bottom reflectance; these were not used in further analysis.

\section{References}

IOCCG (2006). Remote Sensing of Inherent Optical Properties: Fundamentals, Tests of Algorithms, and Applications. Lee, Z. P. (ed.), Reports of the International Ocean-Colour Coordinating Group, No. 5, IOCCG, Dartmouth, Canada.

IOCCG report 5. http://www.ioccg.org/groups/lee_data.pdf

\section{$\underline{\text { Section S2 }}$}

Table S2: Comparison statistics between the QAA-V and QAA-v6 algorithms based on simulated Hydrolight $^{\circledR}$ dataset (HL) and in-situ estuarine and near-shore dataset (IES). $\mathrm{N}=$ number of observations, $\mathrm{RMSE}=$ Root mean square error, $\mathrm{MRE}=$ mean relative error.

\begin{tabular}{|c|c|c|c|c|c|c|c|c|c|}
\hline & $\mathbf{N}$ & \multicolumn{2}{|c|}{$\operatorname{BiaSlog} 10\left(m^{-1}\right)$} & \multicolumn{2}{|c|}{$\operatorname{RMSE}_{\log 10}\left(\mathrm{~m}^{-1}\right)$} & \multicolumn{2}{|c|}{ MRE (\%) } & \multicolumn{2}{|c|}{$\mathbf{R}^{2}$} \\
\hline & & QAA-V & QAA-v6 & QAA-V & QAA-v6 & QAA-V & QAA-v6 & QAA-V & $\begin{array}{c}\text { QAA- } \\
\text { v6 }\end{array}$ \\
\hline \multicolumn{10}{|c|}{ Synthetic data } \\
\hline$a_{\text {tnw }} 411$ & 561 & -0.028 & -0.088 & 0.074 & 0.117 & 12.7 & 19.7 & 0.92 & 0.92 \\
\hline atnw 443 & 561 & -0.006 & -0.071 & 0.071 & 0.106 & 12.7 & 17.7 & 0.91 & 0.90 \\
\hline atnw489 & 561 & -0.003 & -0.076 & 0.072 & 0.110 & 13.0 & 18.0 & 0.91 & 0.89 \\
\hline$a_{\text {tnw } 555}$ & 561 & -0.020 & -0.118 & 0.096 & 0.149 & 16.3 & 23.9 & 0.87 & 0.87 \\
\hline bbtnw 411 & 561 & -0.041 & -0.159 & 0.097 & 0.173 & 15.5 & 28.9 & 0.95 & 0.95 \\
\hline$b_{\text {btnw }} 443$ & 561 & -0.025 & -0.130 & 0.086 & 0.152 & 14.4 & 25.3 & 0.95 & 0.94 \\
\hline$b_{\text {btnww }} 489$ & 561 & -0.017 & -0.119 & 0.082 & 0.146 & 14.1 & 23.9 & 0.95 & 0.94 \\
\hline bbtnw555 & 561 & -0.018 & -0.121 & 0.089 & 0.153 & 14.9 & 23.9 & 0.94 & 0.93 \\
\hline \multicolumn{10}{|c|}{ IES dataset (Testing set: $\mathbf{N}=\mathbf{2 1 9}$ ) } \\
\hline atnw 443 & 209 & -0.023 & -0.091 & 0.142 & 0.180 & 22.7 & 25.8 & 0.83 & 0.76 \\
\hline atnw555 & 209 & -0.029 & -0.124 & 0.190 & 0.249 & 34.3 & 47.5 & 0.72 & 0.63 \\
\hline bbtnw 532 & 89 & 0.038 & -0.049 & 0.173 & 0.174 & 26.0 & 34.6 & 0.70 & 0.67 \\
\hline
\end{tabular}

$\underline{\text { Section S3 }}$

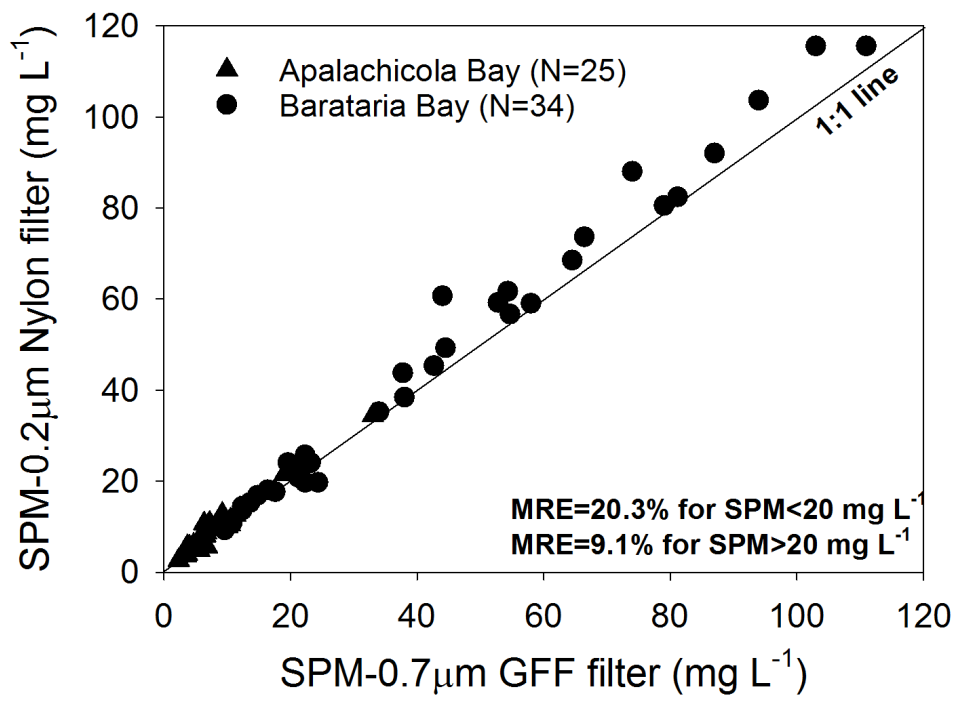

Figure S2: A comparison of SPM concentration obtained with $0.2 \mu \mathrm{m}$ Nylon filter and $0.7 \mu \mathrm{m}$ GFF filters. 


\section{$\underline{\text { Section S4 }}$}
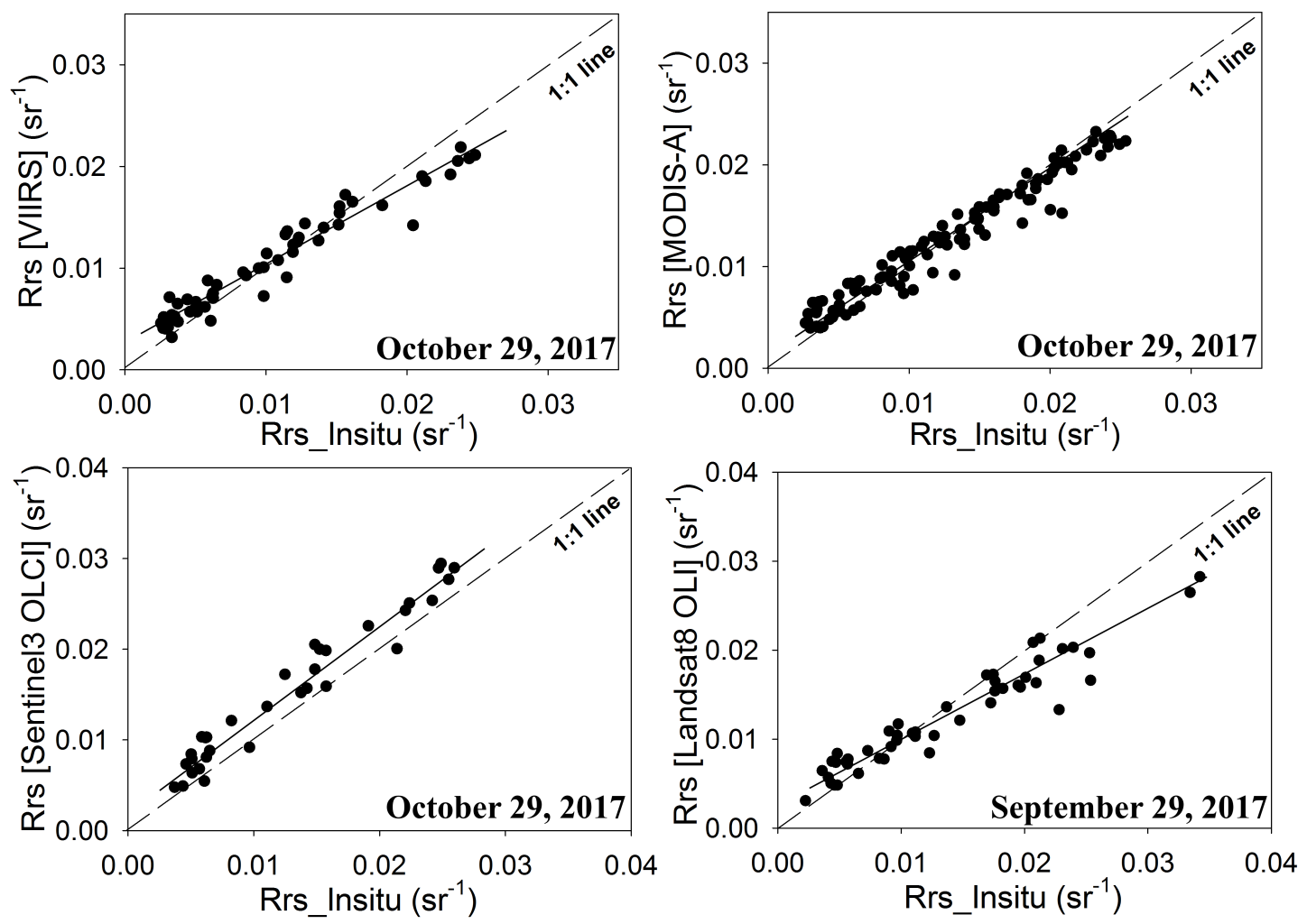

Figure S3: Validation of the atmospheric corrected images that were used for generating maps of $a_{\text {tnw }} 443$ and $b_{\text {tnw }} 470$ in Fig. 10 of the manuscript. 